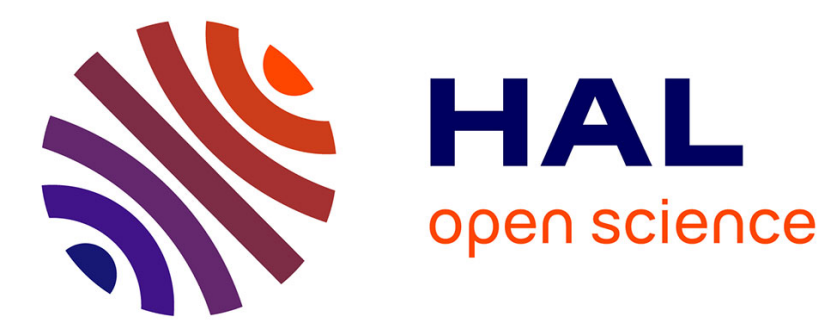

\title{
Predistortion performance considering Peak to Average Power ratio reduction in OFDM context
}

\author{
O. Abel Gouba, Yves Louët
}

\section{To cite this version:}

O. Abel Gouba, Yves Louët. Predistortion performance considering Peak to Average Power ratio reduction in OFDM context. WCNC 2012, Apr 2012, Paris, France. 5 p., 10.1109/WCNC.2012.6214128 . hal-00679272

\section{HAL Id: hal-00679272 \\ https://hal-centralesupelec.archives-ouvertes.fr/hal-00679272}

Submitted on 15 Mar 2012

HAL is a multi-disciplinary open access archive for the deposit and dissemination of scientific research documents, whether they are published or not. The documents may come from teaching and research institutions in France or abroad, or from public or private research centers.
L'archive ouverte pluridisciplinaire HAL, est destinée au dépôt et à la diffusion de documents scientifiques de niveau recherche, publiés ou non, émanant des établissements d'enseignement et de recherche français ou étrangers, des laboratoires publics ou privés. 


\title{
Predistortion Performance considering Peak to Average Power Ratio Reduction in OFDM context
}

\author{
O. Abel GOUBA and Yves LOUËT \\ IETR / SUPELEC, Campus de Rennes \\ Avenue de la Boulaie - CS 47601 \\ F-35576 Cesson-Sevigné cedex, France \\ Email: \{Abel.Gouba, Yves.Louet\}@ supelec.fr
}

\begin{abstract}
Some recent communication systems like DVB-T2 standard set up a PAPR reduction technique followed by a linearization's one. So in such a scenario, the performance of the linearization is influenced undoubtedly by the PAPR reduction method. In this paper, we revisit the EVM metric and evaluate a closed form regarding the performance of both the PAPR reduction technique and the linearization's one. We choose the predistortion as a linearization technique and define a predistortion error. Assuming that the baseband OFDM signal is characterized as a complex Gaussian process, we consider the three top categories of PAPR reduction methods presented in [2] and we first study the distribution of the resulted signal. Then, we derive some theoretical expressions of the first and second order moments of the predistortion error and show that the error depends mainly on the PAPR of the signal after PAPR reduction method and on the predistortion quality. Some simulations compared to our proposed model confirm our results.
\end{abstract}

\section{INTRODUCTION}

Multi-carrier modulations are widely used in wireless and wireline communication systems such as OFDM (Orthogonal Frequency Division Multiplexing). Unfortunately, their implementation is restricted both by the peak signal power also termed as PAPR (Peak-to-Average Power Ratio) and the nonlinearities of the High Power Amplifier (HPA) leading to increase errors, spectral regrowths and reduced power efficiency. This has motivated many works in literature aiming to control the peak power [7]-[11] and the distortions introduced by the HPA [3]-[5]. Two solutions were then proposed, the PAPR reduction and the linearization. The PAPR reduction mainly allows to increase the power efficiency by reducing the dynamic of the signal. This solution include some methods like Amplitude Clipping [7], coding [8], [14], [15], Selected Mapping (SLM) [10], Tone Reservation [11], etc. The linearization techniques compensate the non-linearities of the HPA. They have been in use since many years and include digital predistortion [4]-[6], feedback [5], Linear amplification with non-linear component (LINC) method [3], [5], etc. The large number of PAPR reduction techniques and linearization methods has motivated the publication of a synthesis in [1], [2] gathering methods according to some key criteria.

The performance of PAPR reduction technique or linearization method is measured by a certain number of well known metrics. The most common are the Adjacent Channel Power Ration (ACPR), the Noise Power Ratio (NPR), the Error
Vector Magnitude (EVM) or the Bit Error Rate (BER) [1]. These metrics have been widely measured and studied taking into account the PAPR reduction technique or the linearization method but not both at the same time. However, in recent communication systems, both PAPR reduction and linearization are more and more used. The originality of this study is that it estimates the performance of the linearization taking into account the PAPR reduction effect. The OFDM signal because of it's nature follows a complex Gaussian distribution and its envelope a Rayleigh law [13]. But after PAPR reduction technique, this distribution could be modified. The objective of this paper is to study this new distribution and calculate the predistortion error of the amplified signal considering HPA modeling problems and PAPR.

In the rest of this paper, we study in section II the distribution of the OFDM signal after PAPR reduction considering the three categories of methods detailed in [2]. After describing the system model, we develop in section III some theoretical formulas regarding the first and the second order moments of the predistortion error. Some tests results and analysis are presented afterward.

\section{Signal Distribution AFter PAPR REDUCTION}

Very few publications in literature [12], [13] have studied the distribution of the signal after PAPR reduction in spite of the significant number of proposed techniques [1], [2]. It is well known in literature [13] that after a certain number of subcarriers (64), the OFDM signal is approximated by a complex Gaussian distribution. After PAPR reduction, the distribution could changed. The knowledge of the new distribution of the signal is an important issue for the control of the distortions and the power efficiency.

In this section, we will consider the classification of PAPR reduction methods presented in [1] and updated in [2] where most of important PAPR reduction techniques in literature are classified according to some key criteria such as PAPR reduction gain, downward compatibility, BER degradation, power increase, etc.

\section{A. Recall of PAPR reduction methods classification}

Due to the overgrowing success of the OFDM modulation, many PAPR reduction methods have been proposed since these last ten years. Considering their performance, the authors in 
[1], [2] classified them in three top categories which are coding methods, probabilistic methods and adding signal methods.

Are considered to be adding signal methods, all PAPR reduction methods that can be formulated as $P A P R(X+$ $C)<P A P R(X)$ where $X$ refers to the useful data (in time or in frequency domain) and $C$ the peak canceling signal (in time or in frequency domain) necessary to reduce the initial PAPR. The Bussgang theory allows to show that all distortion methods like clipping [7] and companding [1] can be considered as adding signal methods [2]. Others examples of adding signal method are Tone Reservation, Tone Injection, etc [11].

The idea of probabilistic methods is to make several copies of the initial OFDM signal by modifying the phase, the amplitude and/or the sub-carrier position and then select the one with the lowest PAPR. As a consequence, side information is sent to the receiver for initial signal recovering. Selected Mapping (SLM) [10] or Partial Transmit Sequences (PTS) [9] are some examples of these methods.

The coding methods gather all methods using coding techniques to reduce the PAPR. Reed-Muller codes or Trellisshaping [8], [14], [15] are some examples.

\section{B. Distribution of the signal}

Let's consider the OFDM signal represented by (1) where $f_{k}$ are the sub-carriers frequencies and $N$ their number.

$$
x(t)=\sum_{k=0}^{N-1} X_{k} e^{2 j \pi f_{k} t}, 0 \leq k \leq N-1 .
$$

All the symbols $X_{k}$ are statistically independent identically distributed (i.i.d) complex Gaussian random variables with zero mean and typically MQAM, MPSK or APSK. Thanks to central limit theorem, it has been shown [11], [13] that when the number of sub-carriers $N$ is large, the OFDM signal can be approximated by a Gaussian random processes with zero mean. Therefore, the amplitude of the signal converges to a Rayleigh distribution with $\sigma_{r}$ variance and expressed by (2):

$$
p_{r}(r)=\frac{2 r}{P_{r}} e^{\frac{-r^{2}}{P_{r}}},
$$

with $P_{r}=\sigma_{r}^{2}$ the signal mean power. Our study on the distribution of the signal after PAPR reduction is based on the three categories of methods: coding, probabilistic and adding signal.

Let's first consider the case of coding methods. These methods suppose that it exists a number of structured sequences of symbols $X_{k}$ generating low PAPR time domain symbols $x(t)$. Accordingly to this assumption, a new mapping technique is defined using codewords. So, the symbols $X_{k}$ are no longer independent identically distributed and the resulted signal is not Gaussian anymore but depends on the coding method used. For example, in [14] the authors proposed Golay complementary sequences that generate a constant $3 d B$ PAPR signal whose distribution could not be Gaussian anymore. The major drawback of the coding methods is that the code rate is proportional to $\left(\log _{2} N\right) / N$ [11], [15], consequently for $N$ superior to 32, they are not practical. So we focus our study in the rest of this paper on probabilistic and adding signal methods.

In the case of probabilistic methods, the data symbols $\left\{X_{k}\right\}, 0 \leq k \leq N-1$ are multiplied by a discrete deterministic vector $\left\{V_{k}\right\}, 0 \leq k \leq N-1$ before calculating low PAPR time domain symbol $x(t)$. For SLM [10] or PTS [9], only the phase of the symbols are transformed by a vector expressed by $V_{k}^{p}=e^{j \phi_{k}^{p}}, \phi_{k}^{p} \in[0,2 \pi[, p=0, \ldots, P-1$. Basically, probabilistic methods proceed to a linear transformation and the distribution of the time domain signal after PAPR reduction remains Gaussian with zero mean [16].

The adding signal methods constitute an promising category in spite of their huge complexity and a large number of methods are regularly published [1], [2], [11]. The distribution of the PAPR reduced signal $x(t)+c(t)$ depends of the additive signal $c(t)$. According to the PAPR reduction method, the additive signal $c(t)$ is generated differently and may have different distributions. So, the distribution of the time domain low PAPR signal is not Gaussian anymore.

Because of the large number and the diversity of PAPR reduction methods under adding signal category, an exhaustive study is impossible so we consider for our study, the example of amplitude clipping defined by (3) [7]

$$
f(x)=\left\{\begin{array}{ll}
x & |x| \leq A_{\text {clip }} \\
A_{\text {clip }} e^{j \phi(x)} & |x|>A_{\text {clip }}
\end{array},\right.
$$

where $A_{\text {clip }}$ is the clipping threshold. Amplitude clipping is one of the most used methods of PAPR reduction as its simplicity and its efficiency. The study of the clipped signal shows that its distribution $v(r)$ is given by (4) [13]:

$$
v(r)=p_{r}(r) .1_{r \leq A_{\text {clip }}}+\operatorname{Pr}\{r>A\} . \delta\left(r-A_{\text {clip }}\right) .
$$

where $p_{r}(r)$ is the Probability Density Function (PDF) of the OFDM signal amplitude given by (2) and $\delta(r)$ the Dirac impulse. $\operatorname{Pr}\left\{r>A_{\text {clip }}\right\}$ represents the probability that the signal amplitude $r$ is larger than the clipping threshold $A_{\text {clip }}$ and its expression is:

$$
\operatorname{Pr}\left\{r>A_{\text {clip }}\right\}=\int_{A_{\text {clip }}}^{+\infty} p_{r}(r) d r=e^{\frac{-A_{\text {clip }}^{2}}{P_{r}}} .
$$

\section{PRedistortion PERformance CONSIDERING PAPR REDUCTION}

Most of time, to improve the power efficiency and reduce distortions, both PAPR reduction and linearization techniques are applied in transmission systems. The objective of the predistortion as linearization technique, is to compensate the distortions introduced by the HPA. In the literature, it exists many metrics mentioned above allowing to analyze the performance of the predistortion but they are expressed independently from the PAPR reduction technique and linearization method.

Referring to the system depicted in figure 1, we define a predistortion error in this section and we express the first and second moments order of this error based on some assumptions and the distribution of the signal after PAPR reduction studied in section II. 


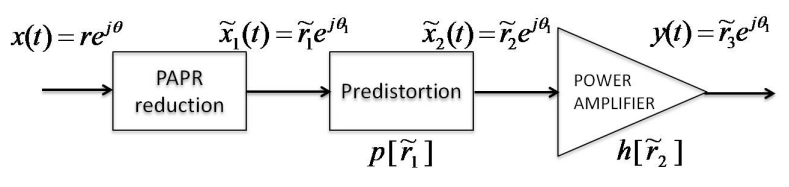

Fig. 1. System model

\section{A. System model and predistortion error definition}

We consider the simplified transmission scheme represented in figure 1 . The OFDM signal $x(t)$ becomes $\tilde{x}_{1}(t)$ after PAPR reduction method and $\tilde{x}_{2}(t)$ after the predistortion technique. The output of the HPA is $y(t)$.

There are two kinds of HPA, the Traveling Wave Tube Amplifier (TWTA) with severe non-linear AM/PM characteristic and the Solid State Power Amplifier (SSPA) with moderate non-linear AM/PM characteristic. In our study, we consider the SSPA amplifier because it is the most used in mobiles applications. Its AM/AM and AM/PM characteristics are expressed by Rapp model [5] as

$$
\begin{aligned}
& h(r)=\frac{r}{\left(1+\left(\frac{r}{A}\right)^{2 b}\right)^{\frac{1}{2 b}}}, \\
& \phi(r)=0,
\end{aligned}
$$

where $A$ is the maximum output amplitude at the saturation point. The parameter $b$ is the "knee factor" that controls the transition smoothness from the linear region to the saturation region. The linearization technique chosen in our study is the predistortion [5]. It consists in applying to the input signal a function $p(r)$ that is exactly the inverse function of the HPA characteristic $h(r)$. So the concatenation of the two will theoretically be equivalent to a linear function, $p(r)=h^{-1}(r)$. The predistortion function $p(r)$ corresponding to the SSPA is equal to

$$
p(r)=\frac{r}{\left(1-\left(\frac{r}{A}\right)^{2 a}\right)^{\frac{1}{2 a}}},
$$

with $a$ the predistorter "knee factor". When $a=b$, a perfect linearization is performed but in practice, this situation is difficult to realize because of modeling problems of the HPA.

In order to evaluate the performance of the predistortion considering a PAPR reduction method, an error $\epsilon$ is defined comparing the amplified signal $y(t)$ to the signal $\tilde{x}_{1}(t)$ before predistortion [6]. AM/PM distortion of the HPA is null as in (6), so $\epsilon\left(\tilde{r}_{1}\right)=\left|\tilde{x}_{1}(t)-y(t)\right|=\left|\tilde{r}_{1}-\tilde{r}_{3}\right|$. This error quantifies the amplitude distortions of the amplified signal depending on the parameters $a, b$ and the dynamic range of the signal amplitude after PAPR reduction. The expression of the error is given by (8).

$$
\begin{aligned}
\epsilon\left(\tilde{r}_{1}\right) & =\left|\tilde{r}_{1}-h\left(p\left(\tilde{r}_{1}\right)\right)\right|, \quad \tilde{r}_{1} \in[0, A[. \\
& =\mid \begin{array}{l}
\tilde{r}_{1}-\frac{\tilde{r}_{1}}{\left(\left(1-\left(\frac{\tilde{r}_{1}}{A}\right)^{2 a}\right)^{\frac{b}{a}}+\left(\frac{\tilde{r}_{1}}{A}\right)^{2 b}\right)^{\frac{1}{2 b}}}
\end{array} .
\end{aligned}
$$

When the predistortion is perfect, $a=b$, the error is null independently from the PAPR reduction method but in real conditions, the error is not null and in the following we study the variations of this error based on the distribution of the input low PAPR signal $\tilde{x}_{1}(t)$. The amplitude $\tilde{r}_{1}$ of the signal $\tilde{x}_{1}(t)$ before predistortion is a random variable so $\epsilon\left(\tilde{r}_{1}\right)$ is also a random variable. As such, the first and second order moments are certain mathematic parameters for its study. Any distribution is characterized by its mean, variance, etc. and the first two order moments are widely used to describe most of them.

However, the error above $\epsilon\left(\tilde{r}_{1}\right)$ may not be expressed in a closed form but it is possible to provide an approximation. One way is to upper bound it. By definition $\tilde{r}_{1} \in[0, A[$. Let's take $u=\left(\frac{\tilde{r}_{1}}{A}\right)^{2 a}$. As a consequence $u \in[0,1[$ and the upper bound gives:

$$
\begin{aligned}
\max _{\tilde{r}_{1}}\left|1-\left(\left(1-\left(\frac{\tilde{r}_{1}}{A}\right)^{2 a}\right)^{\frac{b}{a}}+\left(\frac{\tilde{r}_{1}}{A}\right)^{2 b}\right)^{\frac{-1}{2 b}}\right| \\
=\max _{u}\left|1-\left((1-u)^{\frac{b}{a}}+u^{\frac{b}{a}}\right)^{\frac{-1}{2 b}}\right| \\
=\left|1-\left((1-u)^{\frac{b}{a}}+u^{\frac{b}{a}}\right)^{\frac{-1}{2 b}}\right|_{u=\frac{1}{2}} \\
=\left|1-2^{\frac{b-a}{2 a b}}\right| \\
\text { So } \quad \epsilon\left(\tilde{r}_{1}\right) \leq \tilde{r}_{1}\left|1-2^{\frac{b-a}{2 a b}}\right| .
\end{aligned}
$$

In the rest of this section only the approximation done in (9) will be considered to determine the first and the second order moments of $\epsilon\left(\tilde{r}_{1}\right)$ depending of the distribution of the signal after PAPR reduction.

\section{B. Probabilistic PAPR reduction methods}

The objective in this sub-section is to study the predistortion error defined by (8) when the PAPR reduction method used is a probabilistic one. In this case, the amplitude of the signal follows a Rayleigh distribution with the PDF given in (2). Thanks to that, we can express the first and the second order moments of the predistortion error $\epsilon\left(\tilde{r}_{1}\right)$.

Denoted by $m_{1}$, the first order moment of the predistortion error represents its mean value and it is expressed by:

$$
\begin{aligned}
& m_{1} \triangleq E\left[\epsilon\left(\tilde{r}_{1}\right)\right]=\int_{r_{\min }}^{r_{\max }} \epsilon(r) p_{r}(r) d r \\
& =\int_{r_{\min }}^{r_{\max }}\left[1-\left(\left(1-\left(\frac{r}{A}\right)^{2 a}\right)^{\frac{b}{a}}+\left(\frac{r}{A}\right)^{2 b}\right)^{\frac{-1}{2 b}}\right] \frac{2 r^{2}}{P_{\tilde{r}_{1}}} e^{\frac{-r^{2}}{P_{\tilde{r}_{1}}}} d r
\end{aligned}
$$

where $r_{\min }$ and $r_{\max }$ are respectively the minimum and the maximum values of the reduced PAPR signal amplitude $\tilde{r}_{1} . P_{\tilde{r}_{1}}$ is the mean power of the signal $\tilde{x}_{1}(t)$. Using the approximation of the error given in (9), a close form $m_{1_{1}^{2}} \max$ of the first moment is calculated. Let's consider $\rho=\frac{\tilde{r}_{1}^{2}}{P_{\tilde{r}_{1}}}, \tilde{r}_{1}$ 
defined in $\left[0, A\left[\right.\right.$. We deduct that $\rho$ is in $\left[0, \frac{A^{2}}{P_{\tilde{r}_{1}}}[\right.$. It follows:

$$
\begin{aligned}
& m_{1 \_ \text {max }}=\sqrt{P_{\tilde{r}_{1}}}\left|1-2^{\frac{b-a}{2 a b}}\right|\left[\Gamma_{i n c}\left(\frac{3}{2}, \rho\right)\right]_{\rho=\rho_{\min }}^{\rho=\rho_{\max }} \\
& =\sqrt{P_{\tilde{r}_{1}}}\left|1-2^{\frac{b-a}{2 a b}}\right|\left[\Gamma_{i n c}\left(\frac{3}{2}, \rho_{\max }\right)-\Gamma_{i n c}\left(\frac{3}{2}, \rho_{\min }\right)\right],
\end{aligned}
$$

with $\rho_{\min }$ and $\rho_{\max }$ respectively the minimum and the maximum values of $\rho=\frac{\tilde{r}_{1}^{2}}{P_{\tilde{r}_{1}}}$. $\Gamma_{i n c}$ represents the incomplete gamma function defined as:

$$
\Gamma_{i n c}(z, a)=\int_{0}^{a} x^{z-1} e^{-x} d x .
$$

We can notice that $\rho_{\max }=\frac{r_{\max }^{2}}{P_{\tilde{r}_{1}}}$ is actually the PAPR of the signal $\tilde{x}_{1}(t)$, noted $P A P R_{\tilde{r}_{1}}=\rho_{\max }$. Moreover, the minimum value $r_{\min }$ of the reduced PAPR signal $\tilde{r}_{1}$ is almost null therefore $\rho_{\min }=0$. In this case, (11) becomes:

$$
m_{1 \_ \text {max }}=\sqrt{P_{\tilde{r}_{1}}}\left|1-2^{\frac{b-a}{2 a b}}\right| \Gamma_{i n c}\left(\frac{3}{2}, P A P R_{\tilde{r}_{1}}\right)
$$

The second moment denoted by $m_{2}$ represents the variance of the predistortion error and its expression is written as followed:

$$
m_{2} \triangleq E\left[\left|\epsilon\left(\tilde{r}_{1}\right)\right|^{2}\right]=\int_{r_{\min }}^{r_{\max }}|\epsilon(r)|^{2} p_{r}(r) d r .
$$

By referring to (9), $m_{2}$ is also approximated for $\rho_{\min }=\frac{r_{\min }^{2}}{P_{\tilde{r}_{1}}}$ and $\rho_{\min }=0$. After some maths,

$$
\begin{gathered}
m_{2 \_ \text {max }}=P_{\tilde{r}_{1}}\left[1-2^{\frac{b-a}{2 a b}}\right]^{2}\left[(\rho+1) e^{-\rho}\right]_{\rho=\rho_{\max }}^{\rho=\rho_{\min }} \\
\forall \rho_{\min } \\
=P_{\tilde{r}_{1}}\left[1-2^{\frac{b-a}{2 a b}}\right]^{2}\left[1-\left(P A P R_{\tilde{r}_{1}}+1\right) e^{-P A P R_{\tilde{r}_{1}}}\right] \\
\rho_{\min }=0 .
\end{gathered}
$$

The above equations are defined for $\tilde{x}_{1}(t)$ in $[0, A[$ so $\rho$ is in $\left[0, \frac{A^{2}}{P_{\tilde{r}_{1}}}\left[\right.\right.$. Consequently the PAPR of the signal $\tilde{x}_{1}(t)$, $P A P R_{\tilde{r}_{1}}=\rho_{\max }$ is less than to Input Back Off (IBO) of the HPA, $I B O=\frac{A^{2}}{P_{\tilde{r}_{1}}}$. Hence, from (13) and (16) we show that the predistortion error depends on the PAPR and the IBO, that explains the importance of the PAPR reduction method.

\section{Clipping PAPR reduction method}

In the same way as we proceeded for the probabilistic methods, we are going here to redo the same calculations in the case of the amplitude clipping method which is the adding signal method considered. We calculate the first and the second order moments of the predistortion error using the distribution of the clipped signal given in (4).

The first order moment $m_{1}$ of $\epsilon\left(\tilde{r}_{1}\right)$ when clipping method is used to reduce the PAPR is expressed as follow:

$$
\begin{aligned}
& m_{1} \triangleq E\left[\epsilon\left(\tilde{r}_{1}\right)\right]=\int_{r_{\min }}^{r_{\max }} \epsilon(r) v(r) d r . \\
& =\int_{r_{\text {min }}}^{A_{\text {clip }}} \epsilon(r) p_{r}(r) d r+\epsilon\left(A_{\text {clip }}\right) e^{-\gamma \frac{A_{c l i p}^{2}}{P_{\tilde{r}_{1}}}} .
\end{aligned}
$$

We consider $r_{\max }=A_{\text {clip }}<A . \gamma=1-e^{\frac{-A_{\text {clip }}^{2}}{P r}}$ [7] is the ratio between the mean power $P_{r}$ of the OFDM signal and the mean power $P_{\tilde{r}_{1}}$ of the signal after Amplitude Clipping.

Developing (17) with the approximation done in (9), we obtain $m_{1 \_\max }$ for $\rho_{\min }=\frac{r_{\min }^{2}}{P_{\tilde{r}_{1}}}$ then for $\rho_{\min }=0$.

$$
\begin{aligned}
m_{1 \_ \text {max }}= & \sqrt{P_{r}}\left|1-2^{\frac{b-a}{2 a b}}\right|\left[\Gamma_{i n c}\left(\frac{3}{2}, \gamma \rho\right)\right]_{\rho=\rho_{m i n}}^{\rho=\rho_{c l i p}} \\
& +\epsilon\left(A_{\text {clip }}\right) e^{-\gamma \rho_{\text {clip }}}, \forall \rho_{\min } \\
= & \sqrt{P_{r}}\left|1-2^{\frac{b-a}{2 a b}}\right| \Gamma_{i n c}\left(\frac{3}{2}, \gamma P A P R_{\tilde{r}_{1}}\right) \\
& +\epsilon\left(A_{\text {clip }}\right) e^{-\gamma P A P R_{\tilde{r}_{1}}}, \rho_{\text {min }}=0 .
\end{aligned}
$$

where $\rho_{\text {clip }}=\frac{A_{c l i p}^{2}}{P_{\tilde{r}_{1}}}=P A P R_{\tilde{r}_{1}}$ is the new PAPR of the signal after clipping. This PAPR must be less than the IBO in order to avoid the HPA saturation point.

The second order moment denoted by $m_{2}$ and its approximation $m_{2 \max }$ are calculated too.

$$
\begin{gathered}
m_{2} \triangleq E\left[\left|\epsilon\left(\tilde{r}_{1}\right)\right|^{2}\right]=\int_{r_{\min }}^{r_{\max }}|\epsilon(r)|^{2} v(r) d r \\
m_{2 \_\max }=P_{r}\left[1-2^{\frac{b-a}{2 a b}}\right]^{2}\left[(\gamma \rho+1) e^{-\gamma \rho}\right]_{\rho=\rho_{\text {clip }}}^{\rho=\rho_{\min }} \\
+\left|\epsilon\left(A_{\text {clip }}\right)\right|^{2} e^{-\gamma \rho_{\text {clip }}}, \forall \rho_{\min } \\
=P_{r}\left[1-2^{\left.\frac{b-a}{2 a b}\right]^{2}}\left[1-\left(\gamma P A P R_{\tilde{r}_{1}}+1\right) e^{-\gamma P A P R_{\tilde{r}_{1}}}\right]\right. \\
+\left|\epsilon\left(A_{\text {clip }}\right)\right|^{2} e^{-\gamma P A P R_{\tilde{r}_{1}}, \rho_{\min }=0 .}
\end{gathered}
$$

\section{Simulation results and analysis}

Each simulation in this subsection considers $5.10^{3}$ randomly generated OFDM symbols with 1024 sub-carriers 16QAM modulated. As Probabilistic PAPR reduction method, we choose in the first simulation, Selected Mapping (SLM) [10] for its popularity. For conventional SLM, the phase sequence is created using $\{-1,1,-j, j\}$ and we fix their number to $P=15$. The Input Back Off (IBO) of the HPA is fixed to $7 d B$ and the "knee factor" $b=2$. Due to predistortion modeling as we explained above, $a$ is most of time different from $b$. For several values of $a$, we simulated on figure 2 the predistortion error and plot our proposed theoretical formula expressed in $(11,13,15,16)$. Figure 2 shows that by taking $\rho_{\text {min }}=\frac{r_{\text {min }}^{2}}{P_{\tilde{r}_{1}}}$ or $\rho_{\text {min }}=0$, the curves representing $m_{1 \_\max }$ are similar and both are closed to the simulated $m_{1}$. The plot of the second order moment $m_{2}$ shows the same results.

Figure 3 and 4 present the variations of the first $m_{1}$ and second $m_{2}$ order moments of the predistortion error when amplitude clipping is used as PAPR reduction method. The clipping ratio $C R=\frac{A_{\text {clip }}}{\sqrt{P_{r}}}$ is fixed to $3 d B$ in figure 3 and the $I B O=6 d B$. In figure 4 , we fix $a$ and $b$ and vary the IBO for $C R=3 d B$ and $C R=5 d B$. The comparison of the simulated predistortion errors moments $m_{1}$ and $m_{2}$ on both figures with the proposed approximations $m_{1 \_\max }$ and $m_{2 \_\max }$ shows good agreement as in Selected Mapping case.

The proposed theoretical formulas in (11), (15), (18) and (21) mainly depend from the mean power $P_{r}$, the "knee factor" 


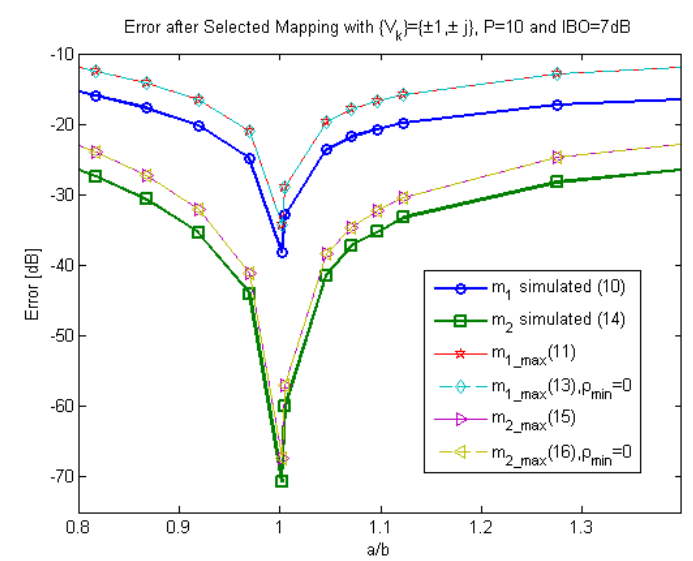

Fig. 2. Predistortion error first and second order moments with Selected Mapping(SLM) as PAPR reduction method

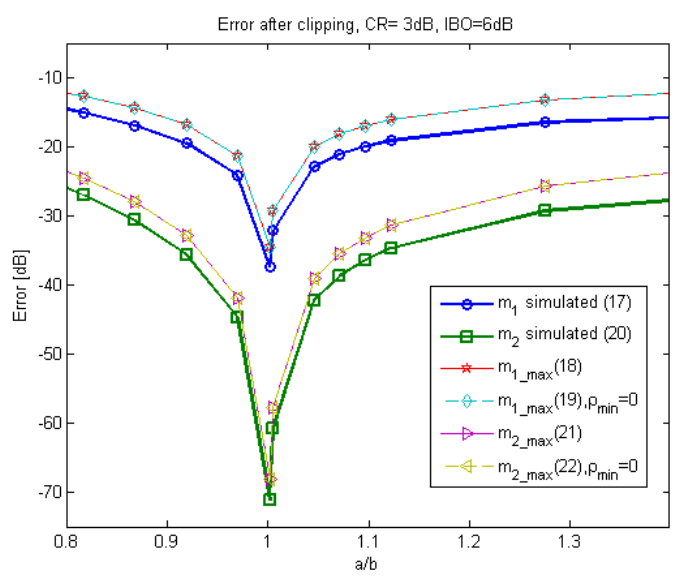

Fig. 3. Predistortion error first and second order moments with amplitude clipping as PAPR reduction method

parameters $a$ and $b$, the $P A P R$ of the signal after the PAPR reduction method and the $I B O$ of the HPA. $I B O$ and $b$ are fixed parameters related to the HPA. In OFDM context, the mean power $P_{r}$ is also known and almost fix. So we can conclude that the key criteria characterizing the performance of the predistortion are the parameter $a$ characterizing the HPA modeling parameter and the PAPR depending on the PAPR reduction method.

\section{CONCLUSION}

Based on systems as shown in figure 1, we analyzed the predistortion performance by studying the first and the second order moments of the predistortion error defined in (8). We derived some closed forms and showed that the performance depends on two parameters: the modeling parameter of the predistorter and the PAPR of the signal after the PAPR reduction method. The simulations validated our theoretical expressions and we noticed that good performance is achieved for a perfect modeling of the preditortion ( $a$ close to $b$ ) and a efficient PAPR reduction method. We focused our study on the predistortion error where any probabilistic PAPR

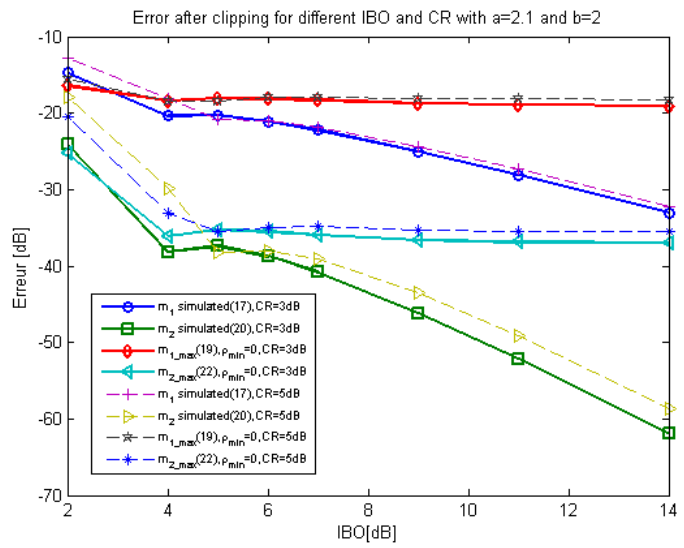

Fig. 4. Predistortion error first and second order moments with amplitude clipping for different $\mathrm{CR}$ and IBO

reduction method or amplitude clipping is applied but the same calculation can be done for the PA efficiency and ACPR or taking into account the memory effects of the HPA. This will be the subject of our future work.

\section{REFERENCES}

[1] Y. Louet and J. Palicot "A classification of methods for efficient power amplification of signals" Annals of Telecommunications, 2008, vol. 63, Issue 7-8, pp 351-368, July/August 2008.

[2] C. Langlais, S. Haddad, Y. Louet and N. Mazouz "Clipping noise mitigation with the capacity approaching FEC codes for PAPR reduction of OFDM signals", in Proc. MC-SS, Herrshing, Germany, 3-4 May 2011.

[3] J-P. Bernoux, J. Palicot, O. Souloumiac, J. Veillard "Dispositif Adaptatif de Prédistorsion pour Amplificateur LINC", GRETSI, 1995, Juan-lesPins, France, 18-21 Sept 1995.

[4] A. Bo, Y. Zhi-Xing, P. Chang-Yong, Z. Tao-Tao, W. Yong and G. Jian-Hua "Improved LUT Technique for HPA Nonlinear Pre-Distortion in OFDM Systems", Wireless Personal Communications 38:495507, 2006.

[5] P. B. Kenington, 'High-linearity RF amplifier design', Artech house, pp. 351-423, 2000.

[6] M.-G. Di Benedetto and P. Mandarini, "A new analog predistortion criterion with application to high efficiency digital radio links", IEEE Transactions on Com., vol. 43 Issue 12, pp. 2966-2974, dec. 1995.

[7] D. Guel et J. Palicot, " Clipping Formulated As an Adding Signal Technique for OFDM Peak Power Reduction ", in Vehicular Technology Conference, 2009. IEEE VTC Spring 2009, Barcelona, Spain, April 2009.

[8] T. A. Wilkinson and A. E. Jones "Minimization of the peak-to-mean envelope power ratio of multicarrier transmission schemes by block coding", in Proc. IEEE VTC, 1995, Chicago, IL, pp 825-829.

[9] Muller SH, Huber JB(1997) "OFDM with reduced peak-to-average power ratio by optimum combination of partial transmit sequences". Electron Lett 33(5):368-369, 1997.

[10] Bäuml R, Fisher R, Huber J "Reducing the PAPR of multicarrier modulation by selecting mapping". E. Lett 32(22):2056-2057, 1996

[11] J. Tellado"Peak to average power reduction for multicarrier modulation." $\mathrm{PhD}$ dissertation, Stanford University, Stanford, USA, 2000.

[12] B. Picinbono "Filtrage préservant les lois marginales", Traitement du signal, 2009, vol. 26, n4, pp. 295-306

[13] P. Banelli, G. Leus, and G. B. Giannakis, "Bayesian Estimation of Clipped Gaussian Processes with Application to OFDM", in Proc. EUSIPCO, vol.1, pp.181-184, Sept. 2002.

[14] Y. Louet 'Etudes et performances des codes de Reed-Muller pour la réduction du facteur de crête dans les modulations OFDM", Ph.D thesis, Rennes I University, Oct 2000.

[15] K. G. Paterson, "Coding techniques for power controlled OFDM, " in Proc IEEE PIMRC, (Boston, MA), pp. 801-805, Sept 1998.

[16] K. Bae and E.J. Powers, "Distribution of envelope power using selected mapping in OFDM systems with nonlinearity", in Proc. IEEE ICASSP, 2008, pp.3065-3068. 